\title{
AVALIAÇÃO LABORATORIAL DO SINAL DE RESSONÂNCIA MAGNÉTICA NUCLEAR
}

Edmilson Helton Rios ${ }^{1}$; Eric Chear Lopes ${ }^{2}$; Irineu Figueiredo ${ }^{1}$; Bernardo Coutinho Camilo dos Santos ${ }^{3}$; Willian Andrighetto Trevizan $^{3}$; Vinicius de França Machado ${ }^{3}$

${ }^{1}$ Observatório Nacional; ${ }^{2}$ Universidade Federal Fluminense; ${ }^{3}$ Cenpes/Petrobras

Copyright 2014, SBGf - Sociedade Brasileira de Geofísica

Este texto foi preparado para a apresentação no VI Simpósio Brasileiro de Geofísica Porto Alegre, 14 a 16 de outubro de 2014. Seu conteúdo foi revisado pelo Comitê Técnico do VI SimBGf, mas não necessariamente representa a opinião da SBGf ou de seus associados. É proibida a reprodução total ou parcial deste material para propósitos comerciais sem prévia autorização da SBGf.

\section{Resumo}

Examinamos o comportamento do sinal de Ressonância Magnética Nuclear (RMN) adquirido em laboratório. Dados de relaxação magnética transversal $\left(T_{2}\right)$ foram analisados tanto no domínio do tempo de decaimento dos ecos (dado bruto), quanto no domínio dos tempos de relaxação transversal (dado invertido). Entre as avaliações realizadas, destacam-se: i) o efeito da relação sinal-ruído; ii) a influência dos parâmetros de inversão (janela de inversão, número de bins e regularização,); iii) a sensibilidade ao volume de fluido e a porosidade; iv) a dependência da viscosidade dos fluidos e salinidade da água; v) a relação com o tamanho de poro. O trabalho contribui para um melhor entendimento do sinal de RMN e suas implicações para o estudo petrofísico de fluidos e interação rocha-fluido.

\section{Introdução}

Quando submetidos a um campo magnético constante, $B_{o}$, núcleos atômicos que possuem momento magnético e spin nuclear diferente de zero, precessam em torno da direção e sentido desse campo na frequência de Larmor, $\omega_{0}=\gamma \cdot B_{0}$, em que $\gamma$ é a constante giromagnética do isótopo em questão. Quanto maior for a magnitude desse campo, maior será a tendência de orientação dos spins para o mesmo sentido e direção de $B_{0}$. Assim, a magnetização resultante do sistema de spins $\mu_{0}$, sob equilíbrio termodinâmico é paralela e diretamente proporcional a $B_{0}$.

Pulsos de rádio frequência (RF) de baixíssimas amplitudes, porém em ressonância com os isótopos de interesse $\left(\omega_{R F}=\omega_{0}\right)$, modificam a orientação de $\mu_{0}$. Sendo $T_{p}$ o tempo de aplicação do pulso e $B_{1}$ a sua amplitude, o ângulo de deflexão é dado por $\Theta=\gamma T_{P} B_{1}$. Uma vez que a perfilagem de poços por RMN emprega baixos campos magnéticos $B_{0}$, estudos petrofísicos laboratoriais são conduzidos em equipamentos de baixa frequência, décimos a poucas unidades de megahertz para 0 hidrogênio. $\mathrm{O}$ isótopo ${ }^{1} \mathrm{H}$ é o mais sensível a $\mathrm{RMN}$, estando presente em abundância nos fluidos de reservatório (água e hidrocarbonetos). É importante frisar que não é possível detectar sinal da matriz sólida nas condições experimentais da perfilagem.

Cessado a atuação de um pulso de RF, a magnetização do sistema retorna ao seu estado anterior de equilíbrio num processo denominado relaxação magnética. Dois processos simultâneos, porém distintos, ocorrem: a relaxação longitudinal ou spin-rede $\left(T_{1}\right)$ e a relaxação transversal ou spin-spin $\left(\mathrm{T}_{2}\right)$. $\mathrm{T}_{1}$ descreve a recuperação da magnetização ao longo do eixo paralelo a $B_{0}$ e está relacionado à troca de energia entre o sistema de spins e a rede. $T_{2}$ descreve a redução da magnetização no plano transversal e está associada à perda de coerência de fase dos spins. Embora este trabalho seja focado em $\mathrm{T}_{2}$, ambos os processos de relaxação podem ser utilizados para estudos de fluidos e interação rocha-fluido.

A curva de $T_{2}$ é obtida através de uma técnica denominada CPMG, a qual consiste na aplicação de um pulso de $90^{\circ}$ seguido por um trem de pulsos de $180^{\circ}$, Figura 1. Enquanto o primeiro pulso deflete a magnetização para o plano transversal da bobina de detecção, os pulsos subsequentes refocalizam o sinal gerando um eco de spin a cada intervalo $2 \tau$ (=TE, tempo entre ecos). A técnica retarda a perda irreversível de coerência de fase dos spins, possibilitando a medida completa da curva de relaxação transversal $I\left(t_{i}\right)$, composta pelos pontos (SI) adquiridos no centro de cada eco. Antes da aplicação da sequência de pulsos, um tempo de espera TW deve ser considerado para que a magnetização do sistema retorne ao equilíbrio atingindo seu valor máximo.

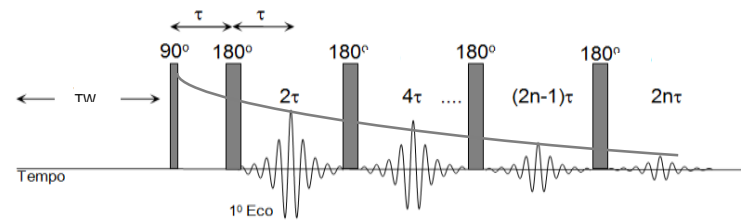

Figura 1 - Representação da sequência de pulsos CPMG, alguns ecos de spin formados e a curva de relaxação transversal $\left(T_{2}\right)$ resultante.

No contexto petrofísico, em que diferentes tipos de fluidos podem estar confinados no meio poroso e interagir com a superfície sólida da matriz, múltiplas contribuições compõem a curva de relaxação transversal. Por isso, as amplitudes do trem de ecos $I\left(t_{i}\right)$ são ajustadas por uma somatória de exponenciais:

$$
I\left(t_{i}\right)=\sum_{j}^{m} A\left(T_{2 j}\right) e^{-\frac{t_{i}}{T_{2 j}}},
$$

em que $A\left(T_{2 j}\right)$ é a amplitude associada a exponencial descrita pela taxa de relaxação $1 / T_{2 j}$. A curva dada pelas amplitudes $A\left(T_{2 j}\right)$ é chamada de distribuição de $T_{2}$ e é 
obtida invertendo-se numericamente a equação 1 através de minimização do erro $\left(\chi^{2}\right)$ entre 0 dado bruto e 0 ajustado. Entretanto, este problema inverso é mal posto, ou seja, pequenas variações em $I\left(t_{j}\right)$, geradas por ruído aleatório, podem levar a soluções completamente diferentes. O mal condicionamento do problema é contornado através da introdução de um termo de regularização na função erro, que no software utilizado tem a forma:

$$
\begin{aligned}
\chi^{2} & =\sum_{i}^{n}\left(I\left(t_{i}\right)-\sum_{j}^{m} A\left(T_{2 j}\right) e^{-\frac{t_{i}}{T_{2 j}}}\right)^{2} \\
& +\alpha \sum_{j}^{m}\left(2 A\left(T_{2 j}\right)-A\left(T_{2(j-1)}\right)-A\left(T_{2(j+1)}\right)\right)^{2},
\end{aligned}
$$

em que "n" e "m" são número de pontos ou bins, respectivamente, do decaimento e da distribuição; e $\alpha$ é o parâmetro de regularização capaz de controlar o quanto o dado deve ser ajustado ou suavizado. É com base na distribuição de $\mathrm{T}_{2}$ que muitas propriedades petrofísicas podem ser indiretamente estudadas.

\section{Resultados}

\subsection{Relação sinal-ruído e inversão}

As medidas de RMN desse trabalho foram realizadas em um equipamento de RMN de bancada do tipo Maran DRX-HF2/50 (Oxford Instruments Ltd, Oxford, UK) com eletrônica digital. O espectrômetro/relaxômetro é equipado com uma sonda de rádio frequencia de $50 \mathrm{~mm}$ de diâmetro, a qual possui uma homogeneidade de campo em torno de $50 \mathrm{~mm}$ na parte central da bobina. Utilizou-se o máximo valor de ganho do receptor para todas as medidas, uma vez que os volumes de fluido investigados são pequenos em comparação ao volume sensível da bobina (baixo fator de preenchimento).

Figura 2 mostra a curva de relaxação transversal $\left(\mathrm{T}_{2}\right)$ para ${ }^{1} \mathrm{H}$ de $1 \mathrm{~cm}^{3}$ de água adquirida sob uma frequência de $2 \mathrm{MHz}$. O dado bruto de RMN é obtido através de dois canais, o real e o imaginário, sendo que ambos possuem informação e ruído, Fig. 2a. O primeiro processamento do dado laboratorial consiste na obtenção de uma média dos pontos (SI) adquiridos no centro de cada eco e a rotação de fase, a qual leva toda informação para apenas um canal, Fig. 2b. As amplitudes estão em unidades arbitrárias do equipamento e são dependentes de parâmetros instrumentais tais como filtro digital e ganho da bobina receptora. A RMN de baixo campo mede sinais inerentemente fracos, sendo necessárias múltiplas aquisições ou scans (NS) para que a razão sinal-ruído (SNR) do dado seja amplificada. Figura 3 compara o dado pré-processado com três diferentes números de aquisição.Como o ruído é aleatório, o sinal aumenta numa proporção maior, Fig.4a.

É importante enfatizar que o número de aquisições necessário para se obter uma boa razão sinal-ruído dependerá do fator de preenchimento da bobina. No experimento realizado, $1 \mathrm{~cm}^{3}$ de água é um volume extremamente pequeno para a dimensão da bobina disponível, por isso muitas médias foram necessárias. Na perfilagem, o volume sensível da bobina detectora é projetado para parede do poço, de modo que todo fluido contido nesse volume contribui com o sinal. Outra diferença importante é que as aquisições (ou stacking) não são estacionárias, o que reduz a resolução vertical da medida de campo.

a)
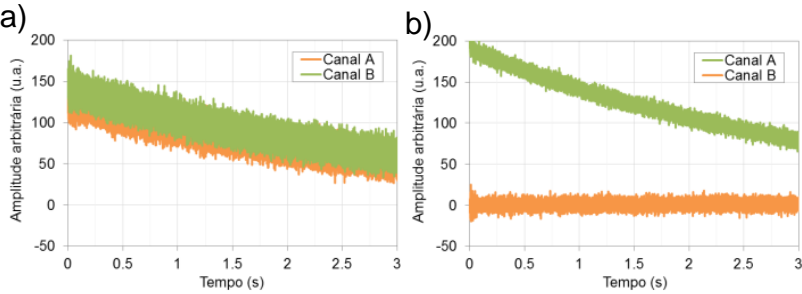

Figura 2 - a) dado bruto de relaxação transversal ( $T E=0,2 \mathrm{~ms}, S I=4, n=74757, T W=20 \mathrm{~s}, N S=128$ ) para $1 \mathrm{~cm}^{3}$ de água. b) dado após médias de $S I$ e rotação de fase (pré-processamento).

Figura 4b. mostra a qualidade do ajuste multiexponencial (Eq. 1) para o decaimento de melhor razão sinal ruído (NS128). Para diminuir os cálculos da inversão, o número de pontos do decaimento foi reduzido de 74757 para 128 através de médias. Apresentando o tempo em escala logarítmica, nota-se que o erro do ajuste é maior para os primeiros ecos da curva. Figura 5 mostra as distribuições de $\mathrm{T}_{2}$ obtidas com a inversão dos dados brutos da Figura 3. No tempo igual a zero, a amplitude máxima do ajuste da curva de decaimento é igual à soma das amplitudes da distribuição de $\mathrm{T}_{2}: I\left(t_{o}\right)=I_{o}=\sum_{1}^{256} A\left(T_{2}\right)$.
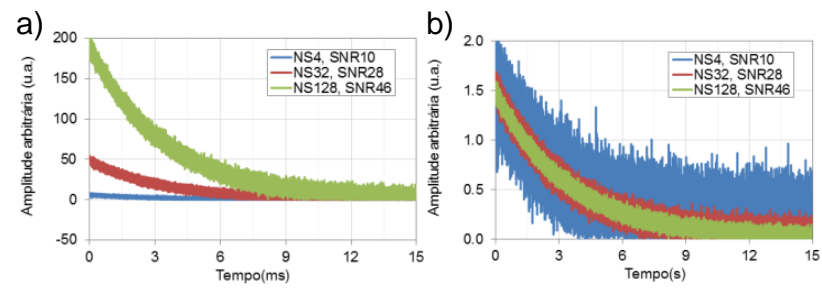

Figura $3-$ a) curvas de relaxação transversal (TE=0,2ms, $S I=1, \quad n=74757, T W=20 \mathrm{~s}$ ) para $1 \mathrm{~cm}^{3}$ de água com número de aquisição variado. b) dado após normalização pelo número de aquisição
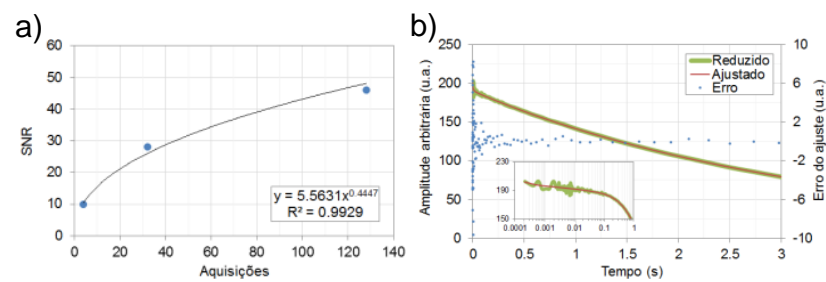

Figura 4 - a) Dependência da razão sinal-ruído com o número de aquisições. b) Curva de decaimento (NS128) após média do número de pontos (redução para 128pts) e respectivo ajuste multiexponencial.

$\mathrm{Na}$ Figura 4, o aumento da relação sinal-ruído melhora a resolução de $\mathrm{T}_{2}$ com $\mathrm{o}$ afinamento das modas. Para que $I_{o}$ mantenha-se constante, as amplitudes dos bins de $\mathrm{T}_{2}$ normalizados sofreram um pequeno aumento. Entretanto, 
o centro da moda principal $(3,5 s)$ praticamente não se altera com a melhora da relação sinal-ruído. Esse é o valor de $\mathrm{T}_{2}$ caso o dado fosse ajustado com somente uma exponencial. Em contrapartida, a posição da moda menor possui uma grande incerteza decorrente de forte influência do ruído no início do trem de ecos.
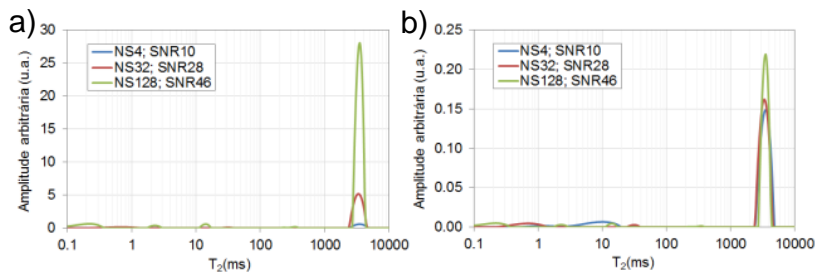

Figura 5 - a) Distribuição de $T_{2}$ (256bins) para $1 \mathrm{~cm}^{3}$ de água com número de aquisição variado. b) dado após normalização pelo número de aquisição.

A janela de inversão foi fixada entre $0,1 \mathrm{~ms}$ e $10 \mathrm{~s}$, com 256 pontos (ou bins) apresentados logaritimicamente. Ela foi propositalmente dilatada para atender a faixa de tempos de relaxação mais comuns em estudos petrofísicos, como veremos nas outras sessões desse trabalho. O efeito da escolha da janela de inversão e do número de bins também foi avaliado nas Figuras $6 \mathrm{a}$ e $6 \mathrm{~b}$, respectivamente. No primeiro caso, embora a janela de inversão tenha sido encurtada, o número de bins foi mantido constante. $O$ aumento da amostragem tanto para essa faixa de $\mathrm{T}_{2}$ quanto para a janela completa, Fig. $6 \mathrm{~b}$, gera um estreitamento da distribuição, aumentando a resolução em $T_{2}$. É importante salientar que, em ambos os casos, a soma das amplitudes dos bins não se alterou e, para que uma comparação pudesse ser feita no mesmo gráfico, as distribuições foram normalizadas pela máxima amplitude.
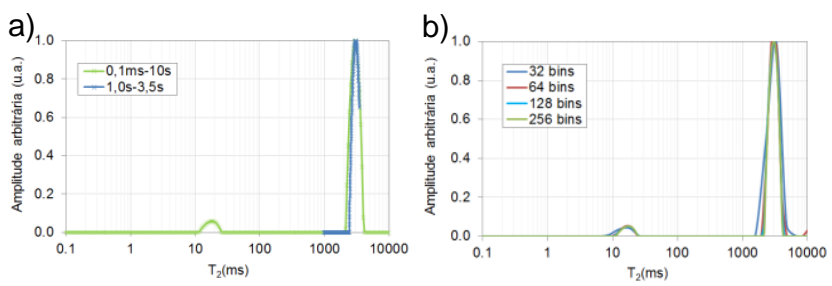

Figura 6 - Distribuições normalizadas de $T_{2}$ para $1 \mathrm{~cm}^{3}$ água $(N S=4)$ variando-se a) janela de aquisição e b) número de bins.

Nas inversões apresentadas nas Figuras 5 e 6 , o parâmetro $\alpha$ foi escolhido automaticamente conforme critério específico do software de inversão WinDxp (Butler et al. 1981), o qual acompanha o equipamento de RMN. $\mathrm{Na}$ Figura 7, demonstramos o efeito do parâmetro de regularização $(\alpha)$ para uma medida de $1 \mathrm{~cm}^{3}$ de água com salinidade de $200 \mathrm{kppm}$. Quanto maior for o $\alpha$, mais larga e incerta é a distribuição de $T_{2}$. Como discutido antes, o sinal a $20 \mathrm{~ms}$ é um artefato que se intensifica com a diminuição do parâmetro alpha, ou seja, com um maior ajuste do dado e, consequentemente, do ruído. Nesse caso, o alpha escolhido pelo software de inversão foi 10 , que representa um balanço entre o excesso de ajuste e suavização do dado bruto.

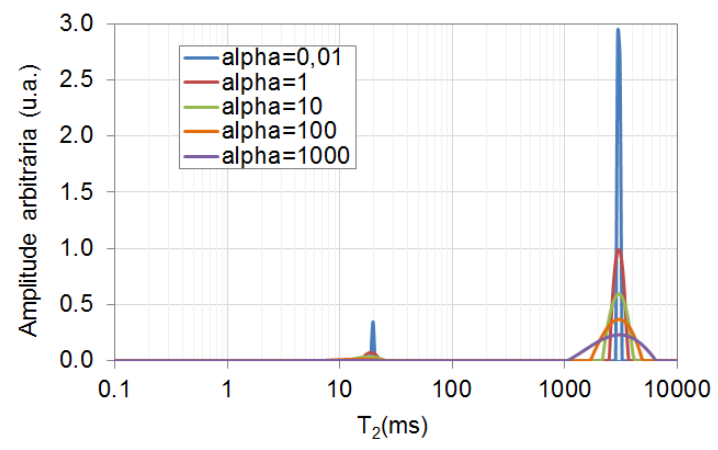

Figura 7 - Distribuição dos tempos de relaxação transversal $T_{2}$ (256bins) para $1 \mathrm{~cm}^{3}$ de água com alpha ( $\alpha$ ) variável.

\subsection{Volume de fluido e porosidade}

O aumento do volume de fluido tem o mesmo efeito que o aumento do número de aquisições uma vez que o sinal adquirido será maior. Figura 8a. mostra que o centro da moda principal de $T_{2}$ não se altera com a variação do volume do fluido, sendo que as modas menores desaparecem da escala com o aumento do SNR. Figura 8b. reforça, primeiramente, que a amplitude inicial do trem de eco é equivalente a soma das amplitudes da distribuição de $\mathrm{T}_{2}$. Na mesma figura, pode-se notar uma excelente linearidade obtida entre o volume de água e a amplitude do sinal de RMN. O volume de fluido é diretamente proporcional à quantidade de hidrogênios presentes, que por sua vez são proporcionais ao sinal detectado na bobina receptora de RMN. Isso implica que, se o sinal de um volume conhecido de fluido é determinado, nas mesmas condições experimentais, um sinal com valor arbitrário informará com bastante precisão o volume de fluido em questão.

Uma vez que os hidrogênios da matriz sólida são insensíveis às condições experimentais empregadas, o sinal de RMN para um fluido confinado em uma rocha saturada é proporcional ao seu volume poroso $\left(\mathrm{V}_{\mathrm{p}}\right)$. Essa condição é válida, como visto anteriormente, para um TW suficientemente longo. Para meios porosos em particular, conforme veremos na seção 2.4, o TE deve ser suficientemente curto para garantir a obtenção do sinal da micro e nanoporosidade.
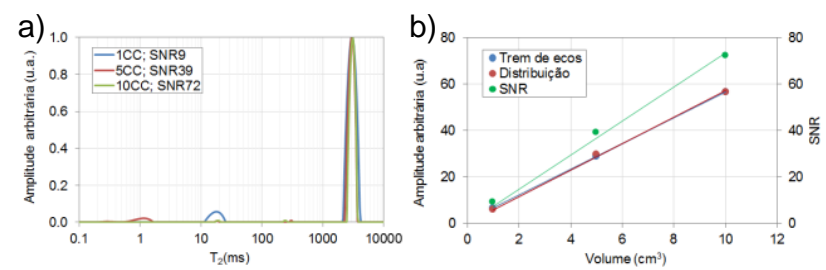

Figura 8 - a) Distribuições normalizadas de $T_{2}$ para diferentes volumes de água (TW=20s, $N S=4)$. b) Relação entre volume de fluido, amplitude total e razão sinalruído.

Em laboratório, o $\mathrm{V}_{\mathrm{p}}$ medido por $\mathrm{RMN}$ pode ser validado com o volume poroso por peso $\left(\mathrm{V}_{\mathrm{pp}}\right)$, que é obtido através 
da diferença entre a massa saturada e seca dividida pela densidade. Sabendo-se o volume total $\left(\mathrm{V}_{\mathrm{t}}\right)$ da amostra, a porosidade é facilmente obtida através da relação $\phi=\mathrm{V}_{\mathrm{p}} / \mathrm{V}_{\mathrm{t}}$. Figura 18 mostra resultados da determinação da porosidade por RMN comparados com porosidades medidas em porosímetro de expansão a gás, técnica padrão para determinação da porosidade em rochas limpas e secas. As nove amostras de rocha utilizadas são de afloramentos areníticos e carbonáticos e foram cortadas em forma de plugue $(3,8 \mathrm{~cm}$ de diâmetro e $5,0 \mathrm{~cm}$ de comprimento), totalmente saturados com água.

Caso o fluido utilizado na calibração do sinal tenha o mesmo volume que o volume total da amostra de rocha, o sinal de RMN pode ser convertido diretamente em porosidade. Esse último método é utilizado na perfilagem visto que o volume de rocha medido na parede do poço é sempre o volume detectável sobre a bobina de superfície da ferramenta. Nesse caso, o sinal do fluido de calibração é associado com uma porosidade equivalente a $100 \%$. Diferentemente de outros perfis de porosidade (nucleares e sônicos, por exemplo) em que calibrações devem ser realizadas para cada tipo de litologia, a porosidade por RMN depende apenas do conteúdo de fluido medido, como comprovado nos resultados da Figura 9.

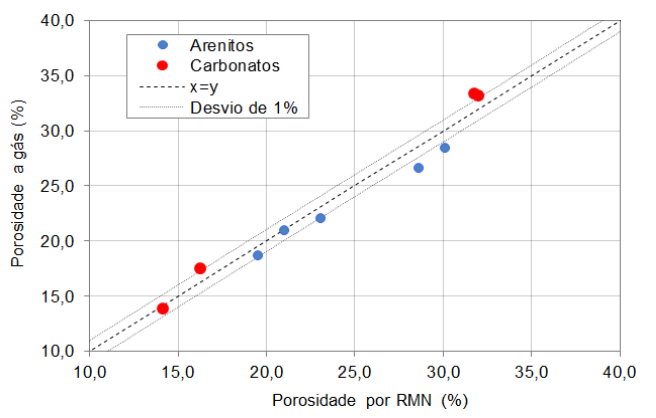

Figura 9 - Comparação entre porosidades do porosímetro a gás e porosidades do RMN (TE=0,2ms, $T R=15 \mathrm{~s})$.

\subsection{Salinidade e viscosidade}

Avaliamos o efeito da salinidade variando a concentração de $\mathrm{NaCl}$ na água. $\mathrm{O}$ aumento da concentração salina gerou uma pequena redução da razão sinal-ruído atribuída ao movimento difusivo dos íons que possuem carga elétrica. Em laboratório, a calibração sinal-volume é realizada sempre com o fluido de mesma salinidade, embora o impacto seja pequeno na determinação do volume. Na Figura $10 \mathrm{a}$, pode-se notar que a variação do pico principal de $\mathrm{T}_{2}$ da água é desprezível e que os picos inferiores são artefatos dependentes da razão sinal-ruído.

$\mathrm{Na}$ Figura 10b., além da distribuição de $\mathrm{T}_{2}$ para a água, mostramos a distribuição para três óleos sintéticos, comercialmente conhecidos como Unipar, Enca e Nujol. A Tabela 1 mostra as principais propriedades reológicas desses fluidos: viscosidade e densidade. Enquanto fluidos leves como a água e o Unipar possuem tempos de relaxação longos e distribuições estreitas, fluidos mais densos e viscosos, geralmente de composição molecular mais variada, tendem a apresentar tempos de relaxação curtos e distribuições largas. O Nujol, por exemplo, possui moléculas com cadeias maiores de menor mobilidade molecular e por isso tempos de relaxação mais curtos.
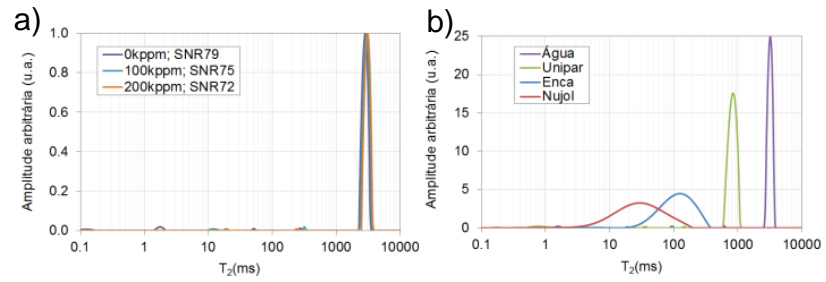

Figura 10 - Distribuição de $T_{2}$ a $30^{\circ} \mathrm{C}\left(10 \mathrm{~cm}^{3}, \mathrm{NS}=4\right)$ para a água com diferentes salidades. b) água e óleos sintéticos com viscosidades diferentes.

Tabela 1 - Propriedades reológicas dos fluidos a $30^{\circ} \mathrm{C}$, obtido com viscosímetro SVM 3000, Anton Paar.

\begin{tabular}{ccc}
\hline Fluido & $\begin{array}{c}\text { Viscosidade } \\
(\mathbf{c P})\end{array}$ & $\begin{array}{c}\text { Densidade } \\
\left(\mathbf{g} / \mathbf{c m}^{3}\right)\end{array}$ \\
\hline Água & 1,0 & 1,00 \\
\hline Unipar & 1,2 & 0,76 \\
\hline Enca & 23,7 & 0,84 \\
\hline Nujol & 138,8 & 0,87 \\
\hline Água & 1,0 & 1,00 \\
\hline
\end{tabular}
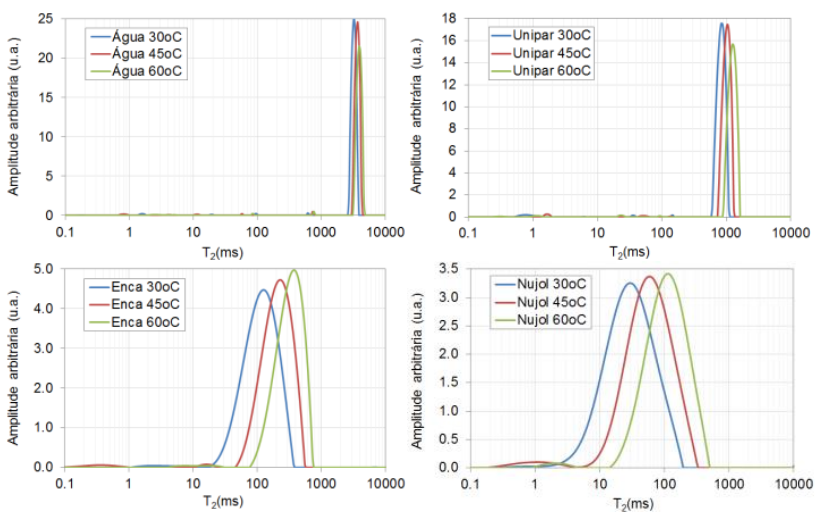

Figura 11 - Efeito da variação de temperatura nos fluidos analisados $\left(10 \mathrm{~cm}^{3}, \mathrm{NS}=4\right)$.

Uma vez que as propriedades reológicas dos fluidos são dependentes da temperatura, medimos as distribuições de $\mathrm{T}_{2}$ para três diferentes temperaturas: 30,45 e $60^{\circ} \mathrm{C}$, Figura 10. O aumento da temperatura causa uma redução da magnetização da amostra devido a um aumento da entropia do sistema. Dessa forma, a máxima amplitude do trem de ecos (ou a soma das amplitudes da distribuição de $\mathrm{T}_{2}$ ) diminui com 0 aumento de temperatura. Tanto o estreitamento quanto o aumento dos valores de $T_{2}$ devem-se a uma diminuição da viscosidade dos fluidos com o aumento da temperatura. 


\subsection{Distribuição de tamanho de poros}

A Figura 12 mostra as distribuições de $T_{2}$ obtidas para a água fora e dentro do meio poroso de nove amostras de rocha, as mesmas utilizadas na seção 2.2. Confinada em um poro, a taxa de relaxação da água bulk $\left(1 / \mathrm{T}_{2 \mathrm{~b}}\right)$ é intensificada devido à interação das moléculas de água com as paredes do poro via difusão. Assim, a taxa efetivamente medida $\left(1 / \mathrm{T}_{2}\right)$ depende da razão superfícievolume do poro (S/V) e da relaxatividade superficial $\left(\rho_{2}\right)$ : $1 / T_{2}=1 / T_{2 b}+\rho_{2}(S / V)$. Se $T_{2 b}$ possuir um valor relativamente alto, como é o caso da água ou Unipar, a taxa de relaxação superficial prevalece, permitindo uma relação indireta dos tempos de relaxação com o tamanho de poro. Assumindo uma geometria esférica, por exemplo, $R_{p}=3 \rho_{2} T_{2}$. Uma vez que o sinal de RMN foi devidamente calibrado para porosidade total (Fig. 9), as amplitudes das distribuições de $T_{2}$ foram apresentadas como porosidade incremental, ou seja, a porosidade associada a cada bin de $T_{2}$, ou família de poro. As propriedades permoporosas dos carbonatos (dolomito e calcário) e arenitos estudados estão descritas na Tabela 2.

Tabela 2 - Propriedades permoporosas das rochas estudadas em permoporosímetro a gás DV-4000, Weatherford.

\begin{tabular}{cccc}
\hline Código & Litologia & $\begin{array}{c}\text { Porosidade } \\
(\%)\end{array}$ & $\begin{array}{c}\text { Permeabilidade } \\
(\mathbf{m D})\end{array}$ \\
\hline SD & Dolomito & 16,3 & 303 \\
\hline WL & Calcário & 32,0 & 1,38 \\
\hline DPL & Calcário & 31,7 & 124 \\
\hline IL & Calcário & 14,2 & 2,02 \\
\hline GBS & Arenito & 19,5 & 111 \\
\hline BBS & Arenito & 20,9 & 320 \\
\hline BS & Arenito & 23,0 & 2600 \\
\hline IGS & Arenito & 28,6 & 4320 \\
\hline IBS & Arenito & 30,1 & 4460 \\
\hline BO & Arenito & 27,7 & 3029 \\
\hline BU & Arenito & 19,7 & 34,8 \\
\hline
\end{tabular}

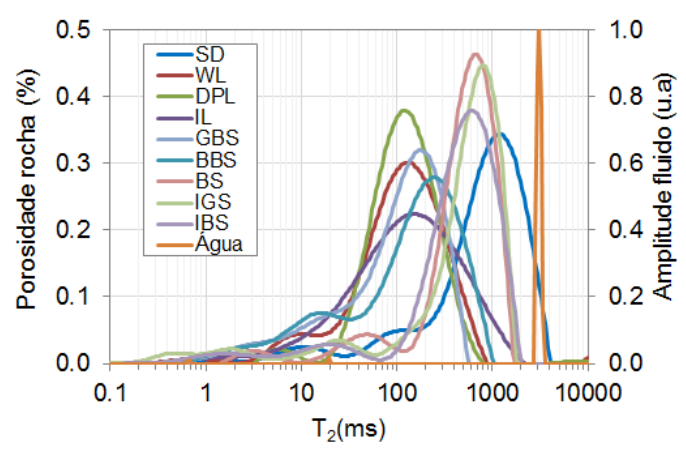

Figura 12 - Distribuição de $T_{2}(T E=200 m s, T W=15 s)$ para água bulk e para nove amostras de rocha totalmente saturadas com água.
Figura 13 mostra a distribuição de $\mathrm{T}_{2}$ para dois arenitos, um de alta (BO) e outro de baixa (BU) permoporosidade. Enquanto BO apresenta uma distribuição de $\mathrm{T}_{2}$ com valores predominantemente altos (macroporosidade), BU possui uma distribuição com tempos mais curtos (micro e mesoporosidade). Na mesma figura é indicada a soma algébrica das distribuições individuais $(\mathrm{BO}+\mathrm{BU})$ e uma nova medida realizada com as duas amostras juntas (BO \& BU). É importante enfatizar que, apesar de se tratar de um método inverso mal posto, a distribuição de $T_{2}$ consegue se manter razoavelmente fiel às distribuições individuais, com maior precisão para as modas de alta amplitude.

A Figura 14 compara a distribuição porosa da amostra SD obtida através de duas técnicas distintas: a RMN e a pressão capilar por injeção de mercúrio $(\mathrm{PcHg})$. O PcHg foi realizado em um fragmento seco do plugue, o qual é submetido à injeção de mercúrio a cada incremento de pressão. Assumindo uma garganta de poro com geometria cilíndrica, a pressão capilar $\left(P_{c}\right)$ é convertida para raio de garganta de poro através da equação de Laplace: $R_{t}=-(2 \sigma \cos \theta) / P_{c}$, onde $\sigma$ é a tensão interfacial e $\theta$ é o ângulo de contato entre ar e mercúrio, ambos tabelados. Para que a comparação pudesse ser realizada, a distribuição de $\mathrm{T}_{2}$ foi convertida para raio de poro, assumindo geometria esférica e relaxatividade superficial igual a $5 \mu \mathrm{m} / \mathrm{s}$.

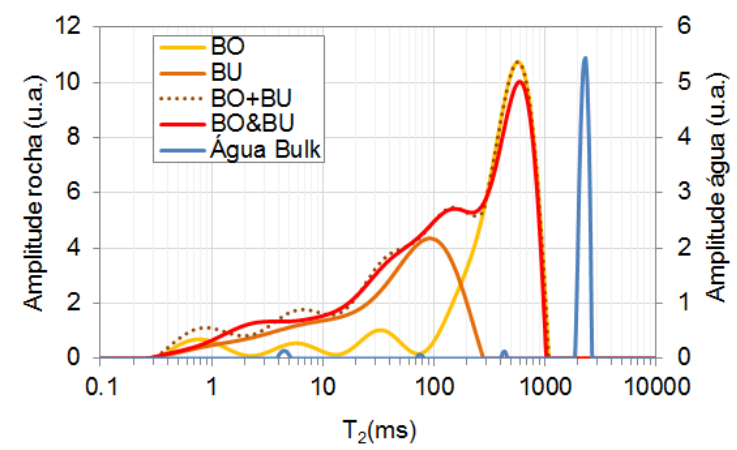

Figura 13 - Distribuição de $T_{2}(T E=200 m s, T W=15 s)$ para água bulk e para duas amostras de rocha totalmente saturadas com água, medidas juntas e separadamente.

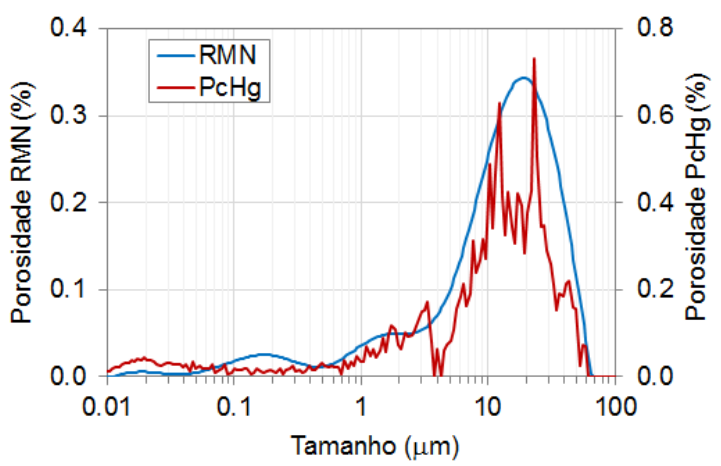

Figura 14 - Distribuição de $T_{2}$ (256pts) da amostra $S D$ comparada com a distribuição de gargantas de poros do PcHg (202pts). 


\section{Conclusões e trabalhos futuros}

O sinal de RMN de baixo campo foi avaliado tanto no domínio dos tempos de decaimento do eco quanto no domínio dos tempos de relaxação transversal $\left(T_{2}\right)$. Constatou-se que: i) a intensidade do sinal é inerentemente baixa, sendo necessário repetir inúmeras vezes a aquisição para se aumentar a razão sinal-ruído do dado; ii) a incerteza em $\mathrm{T}_{2}$ para amplitudes menores é maior e dependente da razão sinal-ruído; iii) A amplitude do sinal de RMN é diretamente proporcional ao volume poroso da rocha e, consequentemente, à porosidade; iv) o tempo de relaxação é dependente das propriedades reológicas do fluido e, no caso da água, pouco sensível a salinidade; v) dependendo da relaxação bulk do fluido saturante, a distribuição de $\mathrm{T}_{2}$ apresenta uma boa relação com a distribuição de tamanho de poros.

Estudos sobre a porosidade e os tempos de relaxação em rochas sob variação dos parâmetros de aquisição (TE e TR) e da influência de gradiente de campo magnético (G) serão realizados dado sua importância para melhor entendimento da resposta do perfil de RMN e sua integração com o dado laboratorial. Uma avaliação sistemática sobre a resposta de sistemas saturantes mais complexos, mono e/ou bifásicos, ainda está em curso. Uma vez que os tempos de relaxação são sensíveis a mobilidade molecular e/ou ao tamanho de poro, eles podem ser ferramentas muito úteis para tipificação e determinação in situ da viscosidade de fluidos, além de estimativa da permeabilidade do meio poroso.

\section{Agradecimentos}

CNPq e PETROBRAS.

\section{Referências}

AMERICAN PETROLEUM INSTITUTE. 1998.
Recommended practices for core analysis.
Recommended Practice RP40, 2nd ed., 220 pp.

Butler, J.P., Reeds J.A., Dawson, S.V., 1981. SIAM J. Numer. Anal. 18, 381.

COATES G, XIAO L \& PRAMMER M. 2001. NMR logging principles and applications. Gulf Professional Publishing, Houston, 234pp.

Darling, T., 2005. Well Logging and Formation Evaluation, Elsevier Science, Burlington, USA, 76-85.

DUNN KJ, BERGMAN, DJ \& LATORRACA GA. 2002. Nuclear Magnetic Resonance Petrophysical and Logging Applications. Elsevier, Amsterdam, 293pp.

ELLIS DW \& SINGER JM. 2008. Well logging for earth scientists. 2nd ed., Springer, Dordrecht, pp 415-478.

Gil VMS \& GERALDES CFGC. 1987. Ressonância Magnética Nuclear: fundamentos, métodos e aplicações. Fundação Calouste Gulbenkian, Lisboa, pp 3-710.
KENYON WE, DAY PI, STRALEY C \& WILLEMSEN JF. 1986. A three-part study of NMR longitudinal relaxation studies of water saturated sandstones, SPE Form. Eval., 3: 662-636.

KLEINBERG RL. 1996. Well logging, Encyclopedia of Nuclear Magnetic Resonance. John Wyley and Sons, New York, pp 4960-4969.

KLEINBERG RL \& JACKSON JA. 2001. An introduction to the history of NMR well logging. Concept. Magnetic. Res., 13(6): 340-342.

LEVITT MH. 2001. Basic principles of NMR spectroscopy. Wiley, Chichester, 686pp.

RIOS et al., 2010. Caracterização petrofísica de rochas reservatório por Ressonância Magnética Nuclear de baixo campo, Paper presented at IV SBGF symposium, Brazil.

RIOS EH. 2011. Caracterização Petrofísica de Rochas Reservatório por RMN: Abordagem Clássica e Modelos Multivariados. Dissertação de Mestrado, Observatório Nacional.

ROSA AJ, CARVALHO RS \& XAVIER JAD. 2006. Engenharia de Reservatórios de Petróleo. Rio de Janeiro: Editora Interciência.

SCHON JH. 2004. Physical properties of rocks: fundamentals and principal of petrophysics. Elsevier, Amsterdam, 583pp.

TIAB D, DONALDSON EC. 2004. Petrophysics: Theory and Practice of Measuring Reservoir Rock and Fluid Transport Properties, 2 ed., Elsevier.

ZHANG Y, XIA P \& YU Y. 2000. Review of Nuclear Magnetic Resonance Magnet for Oil Well Logging. IEEE Trans. Appl. Supercond., 10:763-766. 